\title{
RCS Magnitude Coding for Chipless RFID Based on Depolarizing Tag
}

\author{
O. Rance, R. Siragusa, P. Lemaître-Auger, E. Perret, Senior Member IEEE \\ Université Grenoble Alpes - LCIS \\ 50 Barthélémy de Laffemas, 269002 Valence cedex 9, France \\ olivier.rance@lcis.grenoble-inp.fr
}

\begin{abstract}
An approach for coding information based on the magnitude level of the radar cross section (RCS) of depolarizing chipless tags is proposed for the first time. It is used in combination with the classical frequency coding to increase the coding capacity. The control of the magnitude is based on the polarization mismatch between the tag and the reader antenna and it is fully compatible with a recent technique allowing reliable detection in real environments. The theoretical approach is validated by measurements.

Index Terms - Chipless RFID Tags, Polarization, Radiofrequency identification, Radar cross-sections, Scatterer.
\end{abstract}

\section{INTRODUCTION}

Radiofrequency identification (RFID) is a technology that has emerged in the 40's. Although it is used in many areas, it still didn't manage to replace the optical barcode which remains the preferred candidate for large scale distribution (item tracking). This is mainly due to the low cost and ease of use of the latter. A promising solution to reduce the unit price of a tag is the chipless RFID technology. A chipless tag may be considered as a radar target with a specific electromagnetic signature which is used for coding. Unlike traditional RFID technology, here, the information is encoded directly in the shape of the tag and not within a silicon chip. However, there are two major obstacles that prevent widespread deployment of the chipless RFID technology.

1. The tag's response strongly depends on its environment.

This leads to difficult detection of the tag ID and it limits possible applications in a real environment.

2. The current coding capacity is too small compared to the one obtained with optical barcodes.

The first point was addressed recently by the use of depolarizing tags used with an orthogonal polarization interrogation [1]. Thanks to a calibration process, this technique showed its capacity to detect a unique tag in a real environment and also that a robust detection of a tag applied on metal objects was possible $[1,2]$.

The second point remains problematic but authors generally agree to say that the best coding density is obtained for coding based on frequency [1,2]. The basic principle of frequency coding is to control the presence or absence of a

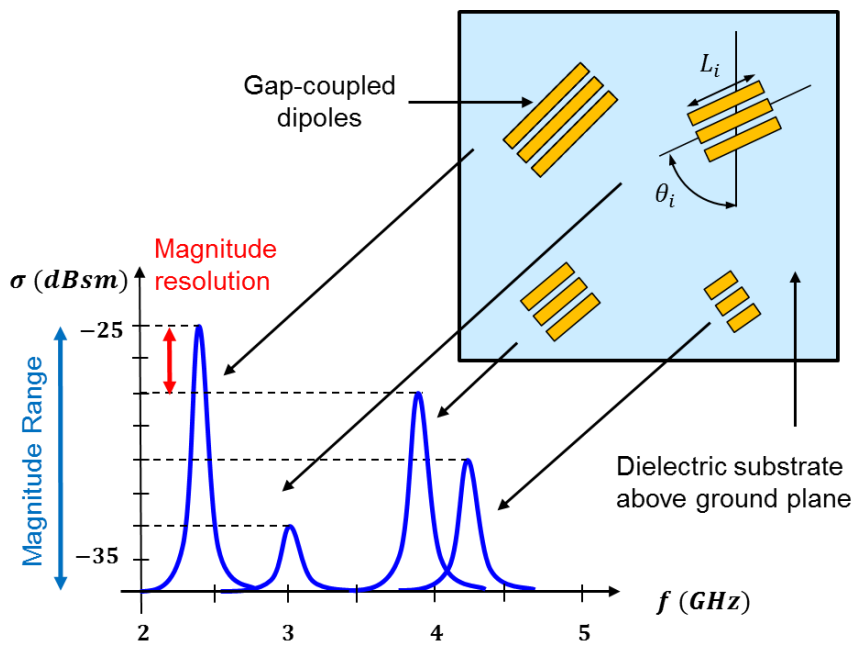

Fig. 1. RCS magnitude coding principle. The tag is composed of resonant scatterers which produces peaks in the scattered spectrum. The information is coded on both the frequency and the RCS magnitude level of each scatterer.

resonance at specific frequencies of the spectrum. Each resonance is associated to a physical scatterer and the coding capacity is then related to the number of resonators present on the tag and to the allowed frequency band. A significant increase can be achieved if each resonator codes several different states. This has been done in frequency with the pulse position modulation approach [3]. It would be interesting to consider a similar approach with scatterers that would however code several RCS magnitude levels in addition to the frequency coding.

In this paper, we are presenting a new coding technique based on the control of the RCS magnitude of depolarizing tags. This technique is completely compatible with the tags and the approach described in [1]. Moreover, it is used in combination with frequency coding and thus it allows an important increase of the coding capacity. Coding information with the RCS magnitude is challenging mainly because the magnitude is highly dependent to the environment (such as distance tag - reader ). To resolve this issue never addressed so far, a specific auto-compensation approach is introduced. 


\section{RCS MAGNITUDE CONTROL}

The principle of RCS magnitude coding is presented in Fig. 1. For each elementary encoding particle (EEP), in addition to the information related to the frequency of occurrence of the peak, the magnitude level of each peak is also coded. The EEP is based on microstrip dipoles [4]. The latter behave like a half wavelength resonator. Their resonnant frequency is set by the strips length $\left(L_{i}\right.$ on Fig. 1). A parametric study has shown that the most suitable approach to adjust the RCS value of the EEP is to rotate the dipoles in the tag's plane $\left(\theta_{i}\right.$ on Fig. 1$)$.

The EEP were optimized in terms of RCS level, selectivity and miniaturization in [1] and the current design is following the same geometry. The tag is composed of four gap-coupled microstrip dipoles. Each one consists of five strips of width $w=2 \mathrm{~mm}$ separated by a gap $g=2 \mathrm{~mm}$, see Fig. 2 . The length of the strips and the corresponding resonant frequencies are given in Table 1. The strips are realized on a Rogers (RO4003C) dielectric substrate with a permittivity of $\varepsilon_{r}=$ 3.55 and a thickness $t=0.8 \mathrm{~mm}$. The EEP are placed over a metallic plate. Each EEP is realized individually in order to modify its respective orientation easily.

The dipoles are sensitive to electromagnetic waves having a linear polarization oriented along the strip direction and show a negligible cross-polarization (direction orthogonal to the strips) response under normal incidence [5]. The interrogating incident plane wave is linearly polarized.

An arbitrary polarized plane wave can always be expressed by the linear combination of two orthogonal fields. A classical way to define the polarization scattered field of a radar target

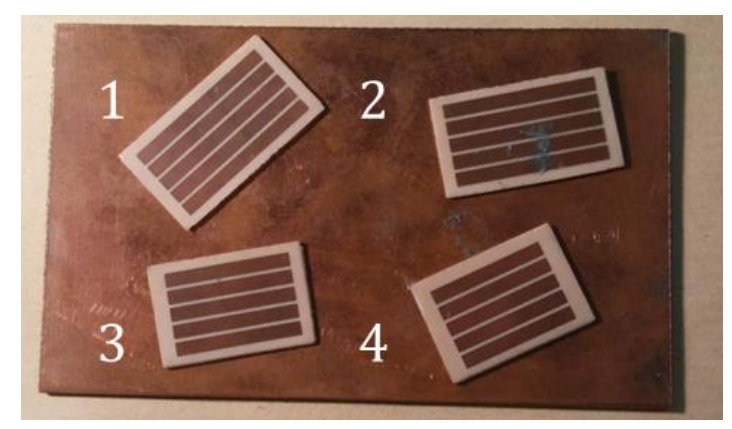

Fig. 2. Picture of a realized tag. Substrate used is Rogers (RO4003C), $\varepsilon_{\mathrm{r}}=3.55$, thickness, $t=0.8 \mathrm{~mm}$.

TABLE I

DIMENSIONS OF THE TAG

\begin{tabular}{|c|c|c|}
\hline$\#$ & $f_{r}(\mathrm{GHz})$ & $L(\mathrm{~mm})$ \\
\hline \hline 1 & 3.4 & 23 \\
\hline 2 & 3.605 & 21.8 \\
\hline 3 & 4.29 & 17.9 \\
\hline 4 & 4.59 & 16.8 \\
\hline
\end{tabular}

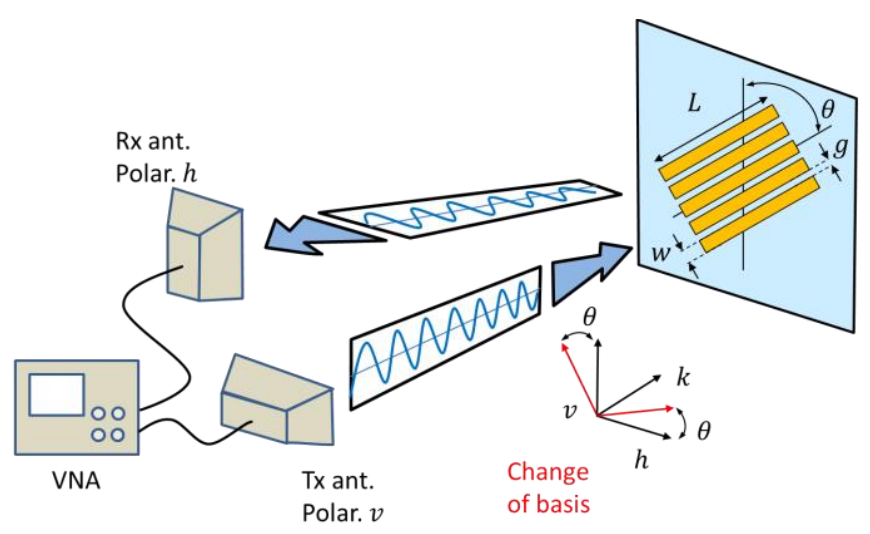

Fig. 3. Orthogonal polarization interrogation. The control of the RCS magnitude is done thanks to the polarization mismatch between the tag and the antennas.

is to use the complex polarimetric RCS matrix [6]:

$$
[\sigma]=\left[\begin{array}{ll}
\sigma_{h h} & \sigma_{h v} \\
\sigma_{v h} & \sigma_{v v}
\end{array}\right] .
$$

For simplicity, the orthogonal linear polarizations, vertical $(v)$ and horizontal $(h)$, are used as a basis for (1). The $v$ polarization is the one of the transmitting antenna while the $h$ polarization is the one of the receiving antenna, see Fig. 3. The measured RCS value thus corresponds to the $\sigma_{v h}$ component. We shall note that for the reference case $\theta=0$, only $\sigma_{h h}$ is non zero.

Applying a rotation to a EEP can be seen like a change-ofbasis (Fig. 3). The RCS in this new basis is expressed as:

$$
\left[\sqrt{\sigma^{\prime}}\right]=[R]^{T}\left[\begin{array}{ll}
\sqrt{\sigma_{h h}} & \sqrt{\sigma_{h v}} \\
\sqrt{\sigma_{v h}} & \sqrt{\sigma_{v v}}
\end{array}\right][R],
$$

where $R$ is a rotation matrix:

$$
[R(\theta)]=\left[\begin{array}{cc}
\cos \theta & -\sin \theta \\
\sin \theta & \cos \theta
\end{array}\right] .
$$

The measured value is then given by:

$$
\sqrt{\sigma_{v h}^{\prime}}=|\cos \theta \sin \theta| \cdot \sqrt{\sigma_{v v}}
$$

It is thus possible to control the RCS magnitude value by varying $\theta$. It is important to note that this coding technique implies that the orientation of the tag to the reader is known. We assume that it is the case in the following. In other cases, it would be possible to recover the value of $\theta$ for most orientations by comparing the values of $\sigma_{v h}^{\prime}$ and $\sigma^{\prime}{ }_{h h}$.

A comparison between theory and measurements is presented on Fig. 4. The tag's response was measured in an anechoic chamber at a distance of $15 \mathrm{~cm}$ from the antennas. The only calibration was an empty measurement (no tag, no object) for isolation between the antennas. A good agreement is observed. The errors for angles under $10^{\circ}$ are due to the sensitivity of (4) which is increasing when $\theta$ approaches zero. To prevent these errors, the angle range is limited from $8^{\circ}$ to $45^{\circ}$ which provides a full scale magnitude range of $6 \mathrm{~dB}$ 
available for coding. The observed deviation of the RCS values is consistent with the uncertainty on $\theta$ evaluated to $1^{\circ}$ in measurements.
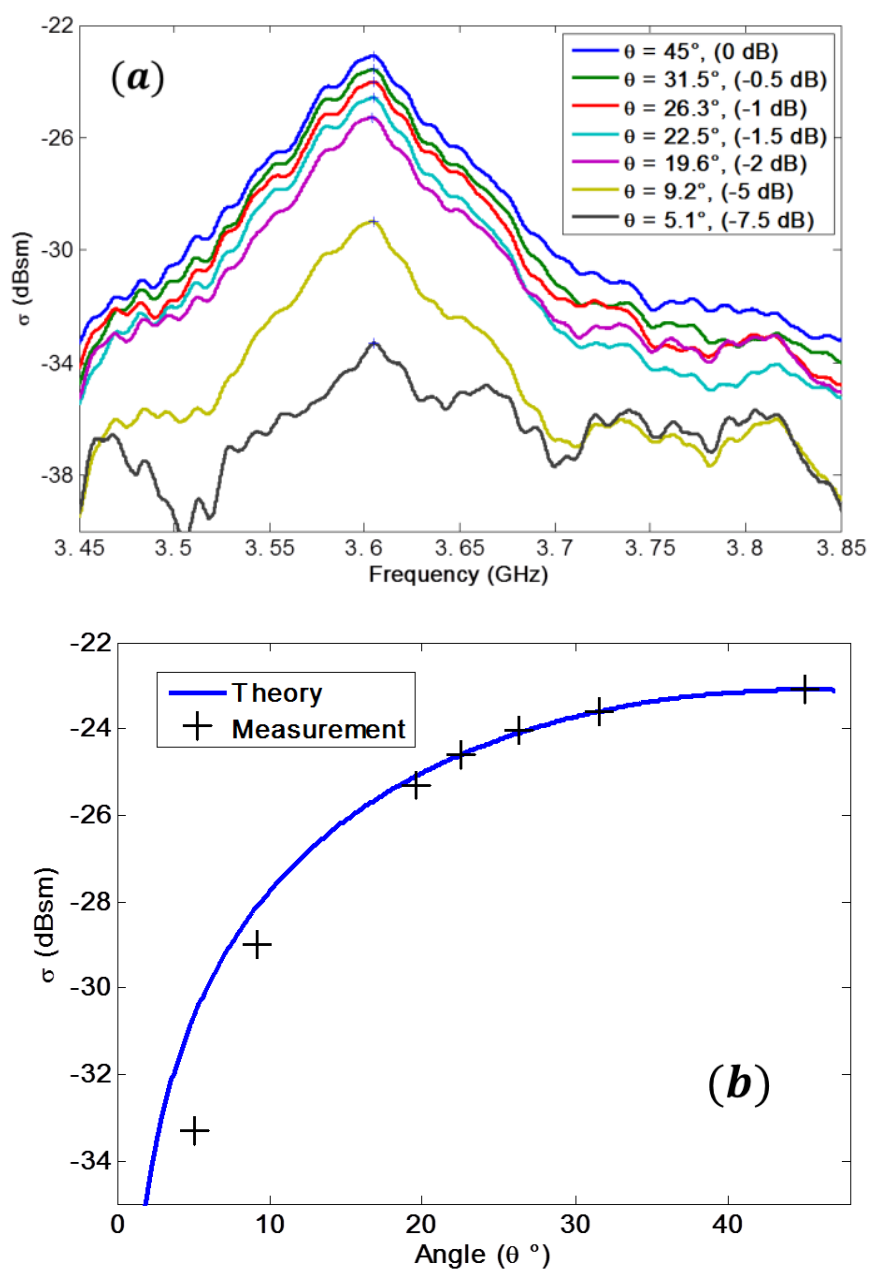

Fig. 4. (a) Measurement of the RCS magnitude of the peak $n^{\circ} 2$ of the tag of Fig. 2 for different values of angle $\theta$. (b) Comparison between the measured and the calculated magnitude using (4).

\section{Measurement Result}

The measured quantity is the backscattered power received by the receiving antenna. If no calibration is done, the backscattered power to the antenna depends on the distance due to free space loss which can lead to erroneous detection of the magnitude. The radar equation shows that the distance induces a translation of the dB-representation of the RCS over the whole frequency band. This phenomenon is represented by measurement results on Fig. 5. As it can be seen, the relative level of the peaks is similar from one distance to another. If one peak is used as a reference scatterer to express the relative value of the others, the correct code can be recovered.

The first peak is taken as the reference and is thus not used to code information. The relative value of the peak 2 with

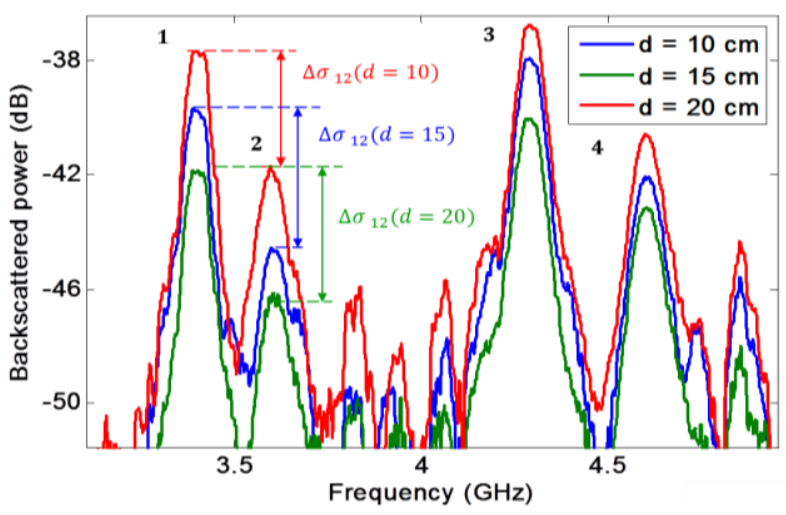

Fig. 5. RCS magnitude level variation with respect to the distance. A relative measurement $\Delta \sigma$ has to be done.

TABLE II

MEASUREMENTS RESUlts

\begin{tabular}{|c|c|c|c|}
\hline$(\mathrm{dB})$ & $\Delta \sigma_{12}$ & $\Delta \sigma_{13}$ & $\Delta \sigma_{14}$ \\
\hline \hline$d=10 \mathrm{~cm}$ & 4 & 1 & 2.9 \\
\hline$d=15 \mathrm{~cm}$ & 4.9 & 1.8 & 2.4 \\
\hline$d=20 \mathrm{~cm}$ & 4.4 & 1.8 & 1.4 \\
\hline \hline Max. error & 0.9 & 0.8 & 1.5 \\
\hline
\end{tabular}

respect to the first one is denoted by $\Delta \sigma_{12}$. The relative values of the peaks are indicated in table 2 and are showing a maximum error for the fourth peak. It enables the assessment of the resolution in magnitude necessary to discriminate one state from another: $\Delta \sigma=1.5 \mathrm{~dB}$. For higher distances, the signal-to-noise ratio will decrease and a higher resolution will be necessary.

The advantage of depolarizing tag is that they can be detected in real environment and in particular when the object to identify is unknown [1]. Measurements at a distance $d=15 \mathrm{~cm}$ have been performed to evaluate the error induced for two objects of different permittivity. The first object was a plate of dielectric of dimension $30 \times 30 \mathrm{~cm}^{2}$. The second was a package of paper of 500 sheets. The measurement results can be observed on Fig. 6. Deviations of $0.5 \mathrm{~dB}$ and $0.9 \mathrm{~dB}$ are

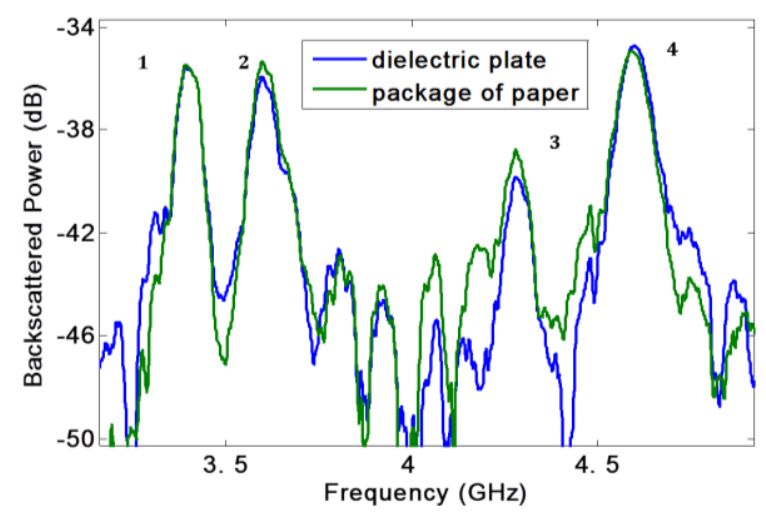

Fig. 6. Measured RCS response of a tag applied on two different objects. 
observed for the peaks 2 and 3 respectively. It is interesting to note from Fig. 4 and Fig. 5 that the peak apex seems much more perturbed by noise than the slopes in its vicinity. This aspect could be exploited to perform more accurate RCS magnitude level detection.

Given the magnitude range and the magnitude resolution, each scatterer can code four different magnitude states. An additional coding capacity of 6 bits is thus obtained.

\section{CONCLUSION}

For the first time the magnitude of the RCS magnitude level was used to code information in chipless RFID. The control of the magnitude is based on the polarization mismatch between the tag and the antennas. This simple approach increased the coding capacity of the initial tag by 6 bits. This coding is fully compatible with a recent technique used for robust detection in real environment.

\section{REFERENCES}

[1] A. Vena, E. Perret and S. Tedjni, "A Depolarizing Chipless RFID Tag for Robust Detection and Its FCC Compliant UWB Reading System," IEEE Trans. Microwave Theory and Tech., vol. 61 , no. 8, pp. 2982-2994, Aug. 2013

[2] F. Costa, S. Genovesi and A. Monorchio, "Chipless RFIDs for Metallic Objects by Using Cross Polarization Encoding," IEEE Trans. Antennas and Prop., vol. 62, no. 8, pp. 4402-4407, Aug. 2014

[3] A. Vena, E. Perret and S. Tedjni, "Design of Compact and AutoCompensated Single-Layer Chipless RFID Tag," IEEE Trans. Microwave Theory and Tech., vol. 60, no. 9, pp. 2913-2924, Sept. 2012

[4] C. A. Balanis, "Microstrip Antenna", in Antenna theory analysis and design, 3rd ed. Ch. 14.

[5] A. Hoorfar, K.C. Gupta and D.C. Chang, "Cross-polarization level in radiation from a microstrip dipole antenna," IEEE Trans. Antennas and Prop., vol. 36, no. 9, pp. 1197-1203, Sep 1988

[6] S. Riegger and W. Wiesbeck, "Wide-band polarimetry and complex radar cross section signatures," Proceedings of the IEEE, vol. 77, no. 5, pp.649-658, May 1989IEEE, vol. 77, no. 5, pp.649-658, May 1989 